\title{
Support Vector Machines for Classification and Quantitative Analysis
}

\author{
Jeffrey M. Davis
}

National Institute of Standards and Technology, 100 Bureau Drive, MS 8371, Gaithersburg, MD

Support vector machines (SVMs) are widely used and broadly applicable machine learning algorithms [1]. Their principal use is for classification algorithms, although they can also be used to develop regressions for predictive modeling. This talk will present the basic theory behind SVMs and show their applications to X-ray microanalysis in the classification of images and in the production of calibration curves for quantitative analysis.

Much of the effort in spectral classification for the microanalysis community has been made in the area of unsupervised classification. In this type of problem, the researcher is attempting to derive the presence of pure phases or compositional classes of materials from the available measured data. The number and compositions of the classes are unknown. However, SVMs are primarily used in classification analysis to solve a group of problems known as "supervised classification" or "supervised learning". For such problems, a "known" data set exists with a series of measured parameters and a sing classification or categorical variable identifying those parameters. For each class the SVM is supposed to identify, a kernel function is created, separating one class from another on the basis of the measured parameters. When new parameters are passed to the SVM, the result is a probabilistic classification.

While quantitative analysis of electron excited X-ray spectra benefits from equations derived from the fundamental interaction of electrons and X-rays, quantitative X-ray excited X-ray fluorescence still uses calibration curves and alpha factor analysis [2]. This is largely due to the fact that absorption and fluorescence corrections for XRF spectra are often significantly larger than in electron excited spectra. The creation of calibration curves requires background modeling, peak fitting and the derivation of $\mathrm{k}$ ratios. Such processing techniques, while accurate, can introduce bias, especially for noisy spectra. By contrast, creating a calibration model using an SVM requires only the known, standard spectra. For quantification, the SRM 482 (Gold-Copper Alloy) was chosen for energy dispersive X-ray fluorescence analysis. Regression modeling using $\mathrm{k}$ ratios and linear least squares fitting were compared against regression models generated using an SVM, which required only the spectrum as an input. The results shown in Figure 2 show the absolute error range from the known values.

For X-ray spectrum based classification, the support vector machine is very well suited for classifying hyperspectral X-ray images. In such images, each pixel is represented by a full X-ray spectrum. The SVM takes, as input, the full 4096 channel X-ray spectrum. It performs no background modeling, peak deconvolution and requires no identification of the elements in the specimen. It references the training spectra provided, and uses those to classify the spectra. Figure 1 shows an example of cement clinker, which is useful because the phases can easily be identified by the backscattered electron image. Although the image was not used to classify the spectra, it is useful as a visual check of the quality of the fitting algorithm. 


\section{References}

1) Cortes, C., Vapnik, V. "Support-vector Networks” Machine Learning 20, 273-297 (1995)

2) De Vries J. and Vrebos B., "XRF Quantification of Infinitely Thick Samples." Handbook of Xray Spectrometry (1993)

\section{BSE Image}

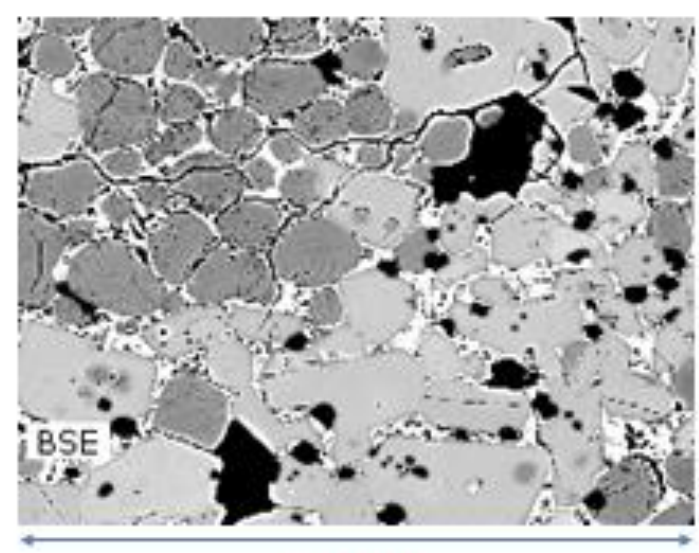

$150 \mu \mathrm{m}$

\section{Classified Image}

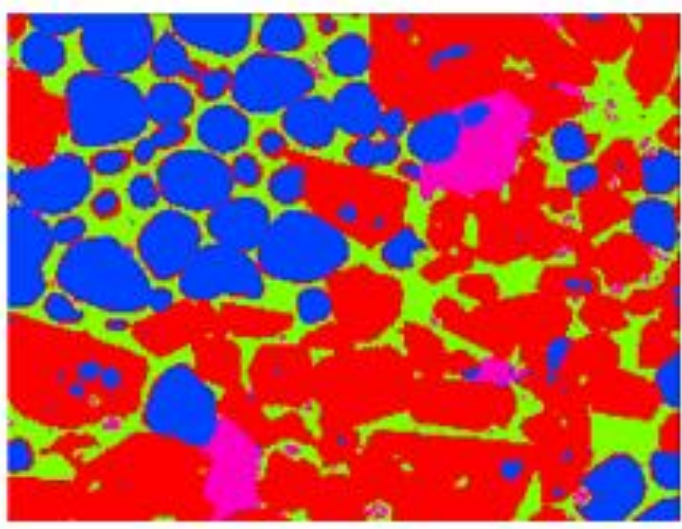

Figure 1: An example of an X-ray image classified by a Support Vector Machine

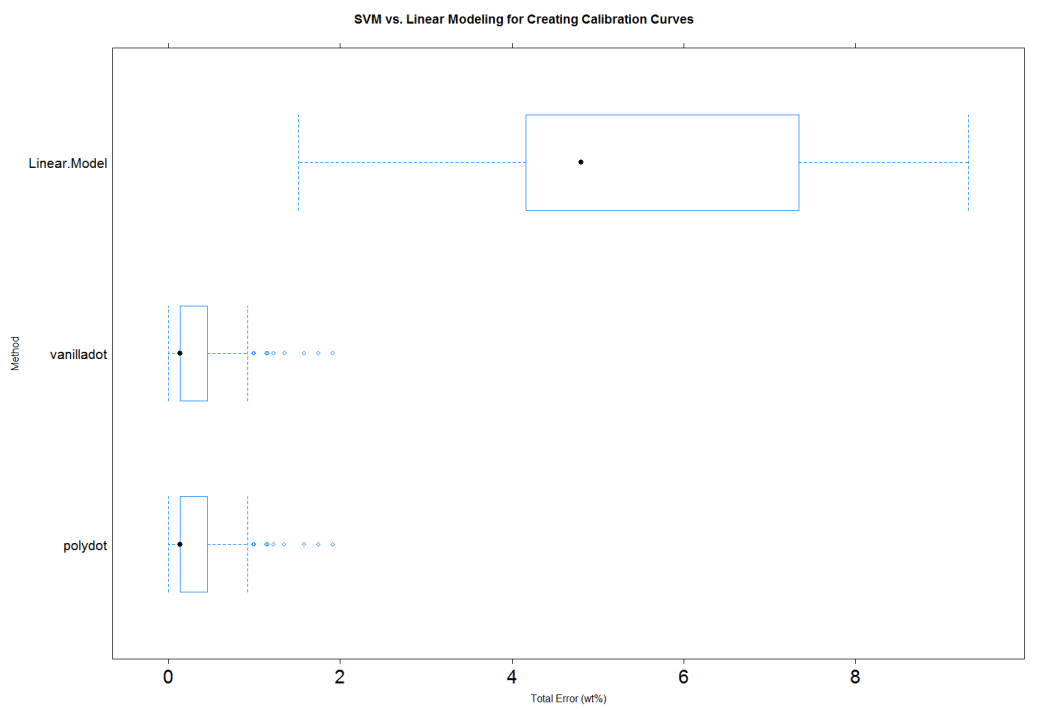

Figure 2: Comparison of errors calculating weight percent composition of copper and gold in SRM 482 